\title{
Lack of riluzole efficacy in the progression of the neurodegenerative phenotype in a new conditional mouse model of striatal degeneration
}

\author{
Grzegorz Kreiner ${ }^{\text {Corresp., }}{ }^{1}$ ， Katarzyna Rafa-Zabłocka ${ }^{1}$ ， Piotr Chmielarz ${ }^{1}$ ， Monika Bagińska ${ }^{1}$, Irena Nalepa ${ }^{1}$ \\ 1 Institute of Pharmacology, Polish Academy of Sciences, Dept. Brain Biochemistry, Kraków, Poland \\ Corresponding Author: Grzegorz Kreiner \\ Email address: kreiner@if-pan.krakow.pl
}

Background. Huntington's disease (HD) is a rare familial autosomal dominant neurodegenerative disorder characterized by progressive degeneration of medium spiny neurons (MSNs) located in the striatum. Currently available treatments of HD are only limited to alleviating symptoms; therefore, high expectations for an effective therapy are associated with potential replacement of lost neurons through stimulation of postnatal neurogenesis. One of the drugs of potential interest for the treatment of HD is riluzole, which may act as a positive modulator of adult neurogenesis, promoting replacement of damaged MSNs. The aim of this study was to evaluate the effects of chronic riluzole treatment on a novel HD-like transgenic mouse model, based on the genetic ablation of the transcription factor TIF-IA. This model is characterized by selective and progressive degeneration of MSNs.

Methods. Selective ablation of TIF-IA in MSNs (TIF-IA ${ }^{\text {DIRCre }}$ mice) was achieved by Cre-based recombination driven by the dopamine 1 receptor (D1R) promoter in the C57BI/6N mouse strain. Riluzole was administered for 14 consecutive days ( $5 \mathrm{mg} / \mathrm{kg}$, i.p.; $1 x$ daily) starting at 6 weeks of age. Behavioral analysis included a motor coordination test performed on 13-week-old animals on an accelerated rotarod (4 to 40 r.p.m.; $5 \mathrm{~min}$ ). To visualize the potential effects of riluzole treatment, the striata of the animals were stained by immunohistochemistry (IHC) and/or immunofluorescence (IF) with Ki67 (marker of proliferating cells), neuronal markers (NeuN, MAP2, DCX), and markers associated with neurodegeneration (GFAP, 8OHdG, FluoroJade C). Additionally, the morphology of dendritic spines of neurons was assessed by a commercially available FD Rapid Golgi Stain ${ }^{\mathrm{TM}}$ Kit.

Results. A comparative analysis of IHC staining patterns with chosen markers for the neurodegeneration process in MSNs did not show an effect of riluzole on delaying the progression of MSN cell death despite an observed enhancement of cell proliferation as visualized by the Ki67 marker. A lack of a riluzole effect was also reflected by the behavioral phenotype associated with MSN degeneration. Moreover, the analysis of dendritic spine morphology did not show differences between mutant and control animals.

Discussion. Despite the observed increase in newborn cells in the subventricular zone (SVZ) after riluzole administration, our study did not show any differences between riluzole-treated and non-treated mutants, revealing a similar extent of the neurodegenerative phenotype evaluated in 13-week-old TIF-IA ${ }^{\text {DIRCre }}$ animals. This could be due to either the treatment paradigm (relatively low dose of riluzole used for this study) or the possibility that the effects were simply too weak to have any functional meaning. Nevertheless, this study is in line with others that question the effectiveness of riluzole in animal models and raise concerns about the utility of this drug due to its rather modest clinical efficacy. 


\section{Author Cover Page}

2

3

4 Lack of riluzole efficacy in the progression of the neurodegenerative phenotype in a new 5 conditional mouse model of striatal degeneration

6

7 Grzegorz Kreiner ${ }^{1 \#}$, Katarzyna Rafa-Zabłocka ${ }^{1}$, Piotr Chmielarz ${ }^{1}$, Monika Bagińska ${ }^{1}$, Irena $8 \quad$ Nalepa $^{1}$

$9{ }^{1}$ Institute of Pharmacology, Polish Academy of Sciences, Dept. Brain Biochemistry,

10 31-343 Kraków, Smętna street 12, Poland

\#Corresponding Author: 


\section{Abstract}

Background. Huntington's disease (HD) is a rare familial autosomal dominant neurodegenerative disorder characterized by progressive degeneration of medium spiny neurons (MSNs) located in the striatum. Currently available treatments of HD are only limited to alleviating symptoms; therefore, high expectations for an effective therapy are associated with potential replacement of lost neurons through stimulation of postnatal neurogenesis. One of the drugs of potential interest for the treatment of HD is riluzole, which may act as a positive modulator of adult neurogenesis, promoting replacement of damaged MSNs. The aim of this study was to evaluate the effects of chronic riluzole treatment on a novel HD-like transgenic mouse model, based on the genetic ablation of the transcription factor TIF-IA. This model is characterized by selective and progressive degeneration of MSNs.

Methods. Selective ablation of TIF-IA in MSNs (TIF-IA ${ }^{\text {D1RCre }}$ mice) was achieved by Cre-based recombination driven by the dopamine 1 receptor (D1R) promoter in the $\mathrm{C} 57 \mathrm{Bl} / 6 \mathrm{~N}$ mouse strain. Riluzole was administered for 14 consecutive days $(5 \mathrm{mg} / \mathrm{kg}$, i.p.; 1x daily) starting at 6 weeks of age. Behavioral analysis included a motor coordination test performed on 13-week-old animals on an accelerated rotarod (4 to 40 r.p.m.; $5 \mathrm{~min}$ ). To visualize the potential effects of riluzole treatment, the striata of the animals were stained by immunohistochemistry (IHC) and/or immunofluorescence (IF) with Ki67 (marker of proliferating cells), neuronal markers (NeuN; microtubule-associated protein-2, MAP2; doublecortin, DCX), and markers associated with neurodegeneration (GFAP, 8OHdG, FluoroJade C). Additionally, the morphology of dendritic spines of neurons was assessed by a commercially available FD Rapid Golgi Stain ${ }^{\mathrm{TM}}$ Kit.

Results. A comparative analysis of IHC staining patterns with chosen markers for the neurodegeneration process in MSNs did not show an effect of riluzole on delaying the progression of MSN cell death despite an observed enhancement of cell proliferation as visualized by the Ki67 marker. A lack of a riluzole effect was also reflected by the behavioral phenotype associated with MSN degeneration. Moreover, the analysis of dendritic spine morphology did not show differences between mutant and control animals.

Discussion. Despite the observed increase in newborn cells in the subventricular zone (SVZ) after riluzole administration, our study did not show any differences between riluzole-treated and 
48 non-treated mutants, revealing a similar extent of the neurodegenerative phenotype evaluated in 49 13-week-old TIF-IA ${ }^{\text {DIRCre }}$ animals. This could be due to either the treatment paradigm (relatively 50 low dose of riluzole used for this study) or the possibility that the effects were simply too weak 51 to have any functional meaning. Nevertheless, this study is in line with others that question the 52 effectiveness of riluzole in animal models and raise concerns about the utility of this drug due to 53 its rather modest clinical efficacy. 


\section{Introduction}

Huntington's disease (HD) is a rare (1:10000) familial autosomal dominant neurodegenerative disorder caused by an expanded stretch of polyglutamine (polyQ) repeats in the protein huntingtin (Hannan 2005) and characterized by progressive degeneration of medium spiny neurons (MSNs) located in the striatum. The disease inevitably culminates with death and cures to at least retard its progression are unavailable so far. Currently available treatments are limited to alleviating some of the symptoms, mainly involuntary movements, associated with the disease. Despite the known origin, there is a lack of understanding of the complex pathogenesis of HD, which affects multiple functions and regulatory pathways, making the development of efficient therapeutics challenging (Kazantsev \& Hersch 2007). Classic pharmacological models of HD are based on applying a neurotoxin, 3-nitropropionic acid (3-NP) (Tunez et al. 2010), however this approach leads to immediate neuronal death, which substantially narrows the opportunity to observe the pathological changes associated with the slow neurodegenerative process. On the other hand, many transgenic animal models of HD, even though created by replicating the same genetic malfunction directly responsible for HD in humans, do not fully recapitulate the HD-like phenotype, including profound neuronal loss (or at least not to the expected extent) (Kreiner 2015).

Designed cell therapies for neurodegenerative diseases are mostly based on the replacement of lost neurons through transplantation or activation of neuronal progenitor cells (Emsley et al. 2005). In rodent models of HD, induced neurogenesis in MSNs is thought to be evoked primarily due to neuronal precursors derived from the subventricular zone (SVZ) of the lateral ventricles. The SVZ represents the largest reservoir of adult stem-like progenitors and in normal conditions gives rise to new olfactory bulb interneurons (Bonfanti \& Peretto 2007). Stimulation of postnatal neurogenesis is being considered as a potential therapeutic target in several neurodegenerative diseases including HD (Abdipranoto et al. 2008; Lindvall \& Kokaia 2010; Ransome et al. 2012). One of the drugs of potential interest for the treatment of HD is riluzole, already approved for the treatment of amyotrophic lateral sclerosis (ALS) (Miller et al. 2012). Riluzole, by interfering with glutamatergic neurotransmission, reduces excitotoxicity and acts as a positive modulator of adult neurogenesis, promoting replacement of damaged MSNs, however, whether it has any clinical meaning remains not clear (Katoh-Semba et al. 2002; Squitieri et al. 2008; Veyrac et al. 2009). It was also shown that riluzole treatment can result in 
87 enhancement of damaged neurite formation potentially leading to functional recovery of motoneurons in rat model of L4-6 root avulsion (Bergerot et al. 2004). Based on experimental data coming from cell and animal research, the classic pharmacological mechanism of its action is related to so-called excitotoxic hypothesis of neurodegeneration. Namely, riluzole can inhibit the release of glutamic acid most likely due to the inactivation of voltage-dependent sodium channels on glutamatergic nerve terminals, as well as activation of a G-protein-dependent signaling pathways (Doble 1996). Another postulated mechanism associated with beneficial role of riluzole application is related to observed increase of serum concentrations of brain-derived neurotrophic factor (BDNF) (Katoh-Semba et al. 2002), which neurotrophic factor is known to be significantly diminished in the brains of HD patients, and its level seems to be correlated with diseases onset progression and severity (Gauthier et al. 2004). Moreover, riluzole was shown to be effective in attenuating several clinically relevant symptoms in a variation of an animal MPTP model representing the early phase of Parkinson's disease (PD) (Verhave et al. 2012). Nevertheless, there are still concerns about its utility due to rather modest clinical efficacy (Miller et al. 2012).

To address this question, we applied a novel approach using a mouse model of HD-like phenotype, based on the activation of an endogenous suicide mechanism achieved by genetic ablation of the transcription factor TIF-IA, an essential regulator of polymerase I activity (Kreiner et al. 2013). Inactivation of TIF-IA blocks the synthesis of ribosomal RNA, leading to nucleolar disruption and p53-mediated apoptosis (Yuan et al. 2005). Loss of TIF-IA in neuronal progenitor cells results in mice born without a brain (Parlato et al. 2008), but when it is lost in mature neurons, the major features of the neurodegenerative process are recapitulated. Namely, inactivation of the TIF-IA gene in striatal MSNs (TIF-IA ${ }^{\text {1RCre }}$ mice) recapitulates the phenotypic alterations associated with selective striatal neurodegeneration (occurring in 13week-old mice), including increased oxidative damage and inflammatory response, finally leading to MSN cell death and resulting in an HD-like phenotype (Kreiner et al. 2013). In particular we have shown that 13 -week-old TIF-IA ${ }^{\text {D1RCre }}$ mice were characterized by profoundly enhanced expression of astro- and microglia markers (GFAP, CD11b), several oxidative stress markers (8-hydroxydeoxyguanosine, $8-\mathrm{OHdG}$; nitrosylated tyrosine, NITT, neuroketals, NK) as well as TUNEL+ cells. The MSNs cells were progressively lost over the time as visualized by NeuN and D1R immunohistochemical stainings. These cellular events were associated with 
118 motor impairment assessed by rotarod and clasping behavior (Kreiner et al. 2013). In contrast to

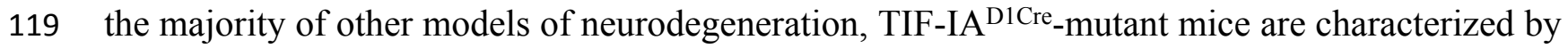

120 the progressive degeneration of targeted neurons over a long period of time (several weeks),

121 mimicking the typical hallmark of the disease (Kreiner et al. 2013).

\section{Materials \& Methods}

123 The summary of experimental design is illustrated on the chart (Fig. 1).

\section{Mice}

125 Selective ablation of TIF-IA in the MSNs (TIF-IA ${ }^{\text {D1RCre }}$ mice) was achieved by Cre/loxP 126 recombination in the $\mathrm{C} 57 \mathrm{Bl} / 6 \mathrm{~N}$ mouse strain. Transgenic mice hosting Cre recombinase under 127 the dopamine 1 receptor (D1R) promoter were crossed with animals harboring the floxed TIF-IA 128 gene as described previously (Kreiner et al. 2013). Mutant TIF-IA ${ }^{\text {D1RCre }}$ mice were kept together 129 with their control (Cre-negative) littermates in self-ventilated cages (Allentown, USA) under 130 standard laboratory conditions (12 h light/dark cycle, food and water ad libitum). This study was 131 carried out in strict accordance with the recommendations in the Guide for the Care and Use of 132 Laboratory Animals of the National Institutes of Health. The protocol for the behavioral study 133 was approved by the Animal Ethical Committee at the Institute of Pharmacology, Polish 134 Academy of Sciences (Permit Number: 951, issued: June 28, 2012).

\section{Drug treatment}

136 After genotyping, the 6-week-old mice were divided into 4 experimental groups: control+VEH 137 (con/VEH), control+RIL (con/RIL), mutant+VEH (mut/VEH), mutant+RIL (mut/RIL), receiving 138 either riluzole (RIL; 5 mg/kg, i.p.; Sigma-Aldrich Chemical Co., St. Louis, USA) or vehicle 139 (VEH; 10\% DMSO) for 14 consecutive days (1x daily). These doses did not influence daily cage 140 normal behavior observed in riluzole and vehicle treated mice.

\section{Behavioral analysis}

142 A coordination test was performed on 13-week-old animals on an accelerated rotarod (Ugo 143 Basile, Italy). The assessment was preceded by training session, 1 day before the experiment (5 144 minutes on the rotating rod, constant speed). During the experiment the time spent on the 145 accelerating rod (4 to 40 r.p.m. within $5 \mathrm{~min}$ ) was measured. Additionally, the weight of the 146 animals was consistently monitored during the time of drug application and on the day before the 147 behavioral test.

\section{Immunohistochemistry}


149 To visualize the potential effects of drug treatment, the striata of animals were subject to post150 mortem staining using immunohistochemistry (IHC) and/or immunofluorescence (IF) with 151 specific markers as described previously (Chmielarz et al. 2013; Kreiner et al. 2013). Briefly, the 152 mice were sacrificed by cervical dislocation, and their brains were excised, fixed overnight in 4\% 153 paraformaldehyde (PFA), dehydrated, embedded in paraffin and sectioned on a rotary microtome 154 on $7 \mu \mathrm{m}$ thick slices. Chosen sections from corresponding regions of the striatum in mutant and 155 control animals were incubated overnight at $4{ }^{\circ} \mathrm{C}$ with primary anti-NeuN (1:500, Millipore; cat. 156 no MAB377), anti-MAP2 (1:1000, Abcam; cat. no ab5392), anti-doublecortin (DCX) (1:100, 157 Abcam; cat. no ab135349), anti-GFAP (1:500, Millipore, cat. no AB5541), and anti-8OHdG 158 (1:200, Millipore; cat. no AB5830) antibodies. Visualization of antigen-bound primary 159 antibodies was carried out using a proper biotinylated secondary antibody together with the 160 Avidin-Biotin Complex (ABC; Vector Laboratories, USA) followed by diaminobenzidine treatment (DAB; Sigma-Aldrich, USA) or an anti-rabbit Alexa-488 or Alexa-594-coupled secondary antibody (Invitrogen, USA). FluoroJade C (Millipore, cat. no AG325) staining was performed according to manufacturer's protocol. Briefly, after deparaffinization and initial incubation in $0.06 \% \mathrm{KMNO} 4$ (10 min.) the slides were rinsed in distilled water and immersed for 10 min. in $0.001 \%$ solution of FluoroJade $\mathrm{C}$ dissolved in $0.1 \%$ acetic acid vehicle.

Quantification of Ki67 expression was done by counting all Ki67-positive cells on adjacent

167

168

169

170

171

172

173

174

175

176

177

178

179

sections from $n=4-6$ animals of each genotype/treatment in a single-blind experiment (an investigator did not know which samples belong to which genotype/treatment).

\section{Dendritic spine morphology}

Morphological analysis of dendritic spines was assessed as described previously (Chmielarz et al. 2015). Briefly, following extraction, the brains were rinsed in distilled water, impregnated with the use of the FD Rapid Golgi Stain ${ }^{\mathrm{TM}}$ Kit (FD NeuroTechnologies, USA), and incubated in 30\% sucrose for 3-7 days. Vibratome (Leica, Germany) sections were cut to $100 \mu \mathrm{m}$ thick and mounted on Super Frost Plus slides (Thermo Scientific, USA) and stained using solutions provided in the kit. The dendritic spines were counted on the dorsal striatum between Bregma 1.1 and 0.0. Dendritic spines were counted on at least $10 \mu \mathrm{M}$ long fragments of $3^{\text {rd }}$ and $4^{\text {th }}$ row dendrites. There were 3 pieces counted from each neuron and 5 neurons counted for each animal. Only completely stained neurons not obscured by neighboring neurons within the striatum were considered. Spine counting and optical imaging were performed by an experimenter blind to the 
180

181

182

183

184

185

186

187

genotype of the animal on a Nikon Eclipse 50i (Nikon, Japan) equipped with a CCD camera connected to a computer equipped with NIS Elements BR 30 software.

\section{Statistical analysis}

Statistical analysis was performed with Graph Pad Prism 5.01. Data were evaluated by 2-way analysis of variance (2-way ANOVA) followed by Bonferroni test for comparison of biologically relevant groups.

\section{Results}

Enhancement of cell proliferation observed in TIF-IA ${ }^{\text {D1Cre }}$ mutant mice and after riluzole treatment.

The expression of Ki67 showed substantial enhancement in the region of the SVZ in nontreated 9-week-old TIF-IA ${ }^{\text {D1Cre }}$ mice and all riluzole-treated animals (Fig. 2A-B). Double immunofluorescent staining revealed that the number of cells labelled with Ki67 (marker of cell proliferation) in SVZ co-localize with the MAP2 (microtubule-associated protein-2, neuronal marker) (Fig. 2C) or doblecortin (DCX) positive cells (Fig. 2D). These cells co-localize with DAPI (marker for nuclear staining) as well.

Lack of riluzole efficacy on progression of MSNs cell death despite enhancement of cell proliferation.

A comparative analysis of immunohistochemical staining patterns with chosen markers characteristic for neurodegenerative process in MSNs (marker for labeling mature neurons, $\mathrm{NeuN}$; an oxidative stress indicator marker, 8-hydroxydeoxyguanosine, 8OHdG; astrocyte marker, GFAP; marker for degenerating neurons, FluoroJade C) did not show any visual differences between 13-week-old mutant TIF-IA ${ }^{\text {1RCre }}$ mice with or without riluzole treatment (Fig. 3A-D). The expression of all of the above-mentioned markers seems to be similar in the riluzole treated and non-treated TIF-IA ${ }^{\text {1RCre }}$-mutant mice, showing comparable enhancement of inflammatory processes, oxidative stress and neuronal loss.

\section{Lack of riluzole efficacy on the behavioral phenotype associated with MSN degeneration.}

Chronic riluzole administration did not prevent impaired motor coordination of 13-week-old mutant TIF-IA ${ }^{\text {D1RCre }}$ mice as demonstrated by the rotarod test (Fig. 4). The riluzole administration had no effect on control animals, while the different effect of the introduced 
209 mutation is reflected in a 2-way ANOVA, which reveals a treatment (riluzole) $x$ genotype

210 interaction for genotype $[\mathrm{F}(131.38)=80.39(\mathrm{p}<0.0001)]$ but not for riluzole itself.

\section{Riluzole does not affect dendritic spine morphology in TIF-IA ${ }^{\text {D1RCre }}$ mice.}

212 Our previous research done on TIF-IA ${ }^{\text {D1RCre }}$ mice clearly showed that although the 213 neurodegeneration (cell loss) is not observed earlier than in 13-week-old animals, some 214 symptoms of cellular impairment can be seen 2-4 weeks in advance (Kreiner et al. 2013). Taking 215 this into account, we checked whether chronic riluzole treatment could have any positive effects 216 on neural cell morphology. Nevertheless, the performed analysis of the morphology of dendritic 217 spines on 9-week-old animals (where no cell loss is observed yet) did not show any differences 218 between mutants and controls (Fig. 5). There was also no effect of riluzole application on 219 dendritic spine morphology in control animals.

220

221

222

223

224

225

226

227

228

229

230

231

232

233

234

235

236

237

238

\section{Discussion}

The objective of this research was supported by preliminary studies, in which we observed an increase in cell proliferation within the SVZ in 9-week-old TIF-IA ${ }^{\text {DIRCre }}$ mice suggesting the existence of ongoing neurogenesis (Fig. 2A-B). This assumption is consistent with other studies reporting increased neurogenesis in other models of progressive neurodegeneration (Luzzati et al. 2011; Nato et al. 2015). This prompted us that the progressive TIF-IA-driven neurodegeneration in these mice offers the unique advantage to study if, in such conditions, the endogenous progenitors potentially involved in putative neuroprotective mechanisms can be modulated by experimental treatments. However, when performed double staining with Ki67 and NeuN within the SVZ in 9-week-old TIF-IA ${ }^{\text {D1RCre }}$ mice, we were not able to find any evidence of co-localization. On the other hand, further immunofluorescent analysis revealed that the number of cells labelled with Ki67 co-localize with the MAP2 or DCX neuronal markers (Fig. 2C-D). This may be explained by the fact that DCX and MAP2 belong to early markers of neuronal maturation, while NeuN is a marker of mature neurons being expressed later on (Sarnat 2013).

To elucidate whether the putative further enhancement of cell proliferation, potentially responsible for evoking adult neurogenesis, induced by chronic riluzole administration can have any positive influence on the progression of the neurodegenerative phenotype observed in TIFIA $^{\text {D1RCre }}$ mice, we evaluated chosen markers of neurodegeneration known to be differentially 
239 expressed in these mice at 13 weeks old, where the cell loss starts to be clearly visible as 240 described previously (Kreiner et al. 2013). Additionally, we also screened their behavioral 241 phenotype by assessing motor coordination. Despite an observed increase in newborn cells in the 242 SVZ after riluzole administration as visualized by Ki-67 staining (an effective marker of 243 proliferating cells (Kee et al. 2002)) (Fig. 2), neither experimental approach showed any 244 differences between riluzole-treated and non-treated mutants, revealing a similar extent of the neurodegenerative phenotype evaluated in 13-week-old animals (Fig. 3-4). The mice were 246 characterized by the same expression of induced-GFAP and $8 \mathrm{OHdG}$ and profoundly reduced 247 staining intensity for $\mathrm{NeuN}$ in the striatum (Fig. 3A-C). The neurodegenerative phenotype was 248 further confirmed by FluoroJade C staining, an effective marker of degenerating neurons 249 (Schmued et al. 2005) (Fig. 3D). This was reflected by the impairment in motor coordination on 250 the rotarod test, and again, no differences were observed between riluzole- and vehicle-treated

251

252

253

254

255

256

257

258

259

260

261

262

263

264

265

266

267

268

269

mutants (Fig. 4). Overall, these experiments did not show any beneficial effects of riluzole administration on the progress of the mutation.

It seemed that further enhancement of this process by riluzole administration can bring considerable benefits in the form of slowing down the progression of the mutation. Our transgenic models based on the conditional ablation of transcription factor TIF-IA have already been positively verified as a possible tool to study the mechanisms of action of other pharmacotherapies. In particular, we showed that the progression of neurodegenerative phenotype in the TIF-IA ${ }^{\text {DATCre }}$ mice (PD model) can be postponed by L-DOPA (Rieker et al. 2011) or reboxetine treatment (Rafa-Zabłocka et al. 2014).

It can be argued that either the treatment paradigm was not appropriate to achieve the expected drug efficacy or the effects were simply too weak to have any functional meaning. Regarding the first issue, the dose of riluzole in chronic experiments performed on rodents does indeed range from 1 to $40 \mathrm{mg} / \mathrm{kg}$ (Besheer et al. 2009; Carbone et al. 2012; Fumagalli et al. 2006; Sepulveda et al. 1999), and is predominantly $20 \mathrm{mg} / \mathrm{kg}$ when used to evoke a neurogenesis response. Therefore, the dose used in our experiment was in the lower range of the therapeutic window. The reason for choosing this particular dose was determined by the lethargy and spastic gait followed by a high mortality rate of the mice treated with 20 and $10 \mathrm{mg} / \mathrm{kg}$. This problem has also been reported by other researchers when rats were treated with similar doses and exhibited locomotor ataxia and lethargy (Kitzman 2009; Simard et al. 2012). We presume that 
270 this phenomenon is associated with the specific mouse strain $(\mathrm{C} 57 \mathrm{Bl} / 6 \mathrm{~N})$ rather than with the 271 introduced mutation since the problem affected both control and TIF-IA ${ }^{\text {DiRCre }}$ mice. 272 Nevertheless, it has to be emphasized that even the dose of $5 \mathrm{mg} / \mathrm{kg}$ was able to induce cell 273 proliferation within the SVZ region as visualized by Ki67 staining (Fig. 2A) and quantified 274 afterwards (Fig. 2B). Moreover, there are existing reports that prove a similar dose to be 275 effective (Kitzman 2009).

276 In addition, in order to evaluate whether riluzole can exert any influence on affected 277 MSNs, we performed a quantitative analysis of dendritic spine morphology at the stage when the

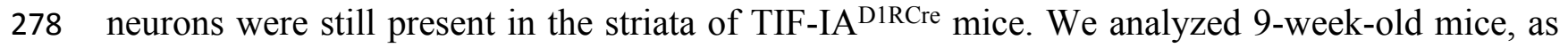
279 this is the stage where no cell loss has been observed but the cascade of molecular events leading 280 to degeneration has already been prompted (Kreiner et al. 2013). Nevertheless, this analysis did 281 not show any changes in dendritic spine morphology (Fig. 5), supporting the observation that 282 riluzole seems to not be effective in the investigated model.

Surprisingly, we were not able to find any abnormalities in the morphology of dendritic 284 spines in the non-treated TIF-IA ${ }^{\text {D1RCre }}$-mutant mice despite the clear neurodegenerative 285 phenotype that has already been documented. This issue has not been addressed in our previous work. However, the occurrence of such changes is not always correlated with neurodegeneration (Dickstein et al. 2010) or may be a subsequent event. On the other hand, lack of spine pathology might also be attributed to the relatively early stage of pathology observed in 9-week-old TIFIA $^{\text {D1RCre }}$ mutants, as other authors have shown that spine pathology was present in late (36-weeks old) (Spires et al. 2004), but not early (20-weeks old) (Nithianantharajah et al. 2009), symptomatic stages of the R6/1 Huntington disease model.

The lack of analysis of other time points (i.e., 16-week-old animals or older) can be regarded as a drawback of the experimental design. This is mainly due to the relatively low number of animals in the cohort, which is restricted by current strict animal welfare regulations. Nevertheless, since the phenotype of riluzole-treated TIF-IA ${ }^{\text {D1RCre }}$ mice is non-distinguishable from untreated mutants (regarding both the behavioral and histological levels) at the age of 13 weeks (where the cells are already starting to degenerate), it would be hard to imagine that any differences would be observed at a later period. Lack of differences at this pivotal stage does not provide any strong support for the investigation of earlier time points, which could have been 300 interesting if we had observed findings differentiating the animals at 13 weeks. However 
301 unlikely, it cannot be excluded that analysis of additional time points in-between 9-th and 13-th

302 week would differentiate the riluzole treated and non-treated animals.

303 In spite of expectations based on previously gathered evidence in preclinical studies and

304 the use of riluzole in clinics for the treatment of ALS, a recent study also yielded disappointing 305 results concerning this drug. Despite being an expensive drug, it does not stop the progression of 306 ALS and is not always well tolerated, making the efficacy of riluzole in the treatment of ALS 307 inconclusive (Ginsberg \& Lowe 2002). Moreover, experiments performed on animal models 308 assessing riluzole as a potential treatment for HD and spinocerebellar ataxia (SCA) had no 309 beneficial effects (Hockly et al. 2006; Schmidt et al. 2016). In clinical trials of anti-HD 310 treatment, there was also no clear neuroprotective effect of riluzole administration, and its effects 311 were narrowed only to reduced chorea (Frank 2014). Thus, our study seems to be in line with 312 others that question the effectiveness of riluzole in animal models and raise concerns about the 313 utility of this drug due to its rather modest clinical efficacy (Limpert et al. 2013).

\section{Conclusions}

Despite an observed increase in newborn cells in the SVZ after riluzole administration, our study did not show any differences between riluzole-treated and non-treated mutants, revealing a similar extent of the neurodegenerative phenotype evaluated in 13-week-old TIFIA $^{\text {D1RCre }}$ animals, a new transgenic model resembling HD-like neurodegeneration. This lack of an observed effect could be due to either the treatment paradigm or the possibility that the effects were simply too weak to have any functional meaning. Nevertheless, this study is in line with others that question the effectiveness of riluzole in animal models and raise concerns about the utility of this drug due to its rather modest clinical efficacy.

\section{Acknowledgements}

We thank Prof. Günther Schütz and Dr. Rosanna Parlato from the German Cancer Research Center (DKFZ, Heidelberg, Germany) for their generous gift of the TIF-IA ${ }^{\text {D1RCre mice. }}$ 
331 Fig. 1. The summary of experimental design. The mice were treated with riluzole $(5 \mathrm{mg} / \mathrm{kg}$, i.p.)

332 starting 6-th week of age. The phenotype of riluzole treated vs non-treated animals was 333 compared at 13-th week of age on behavioral and immunohistochemical level, when the effects 334 of the mutation are clearly manifested in neuronal cell loss and behavioral impairment. Ki67 335 expression and dendritic spines morphology were assessed at 9-th week when cell loss in mutant 336 animals have not yet been observed.

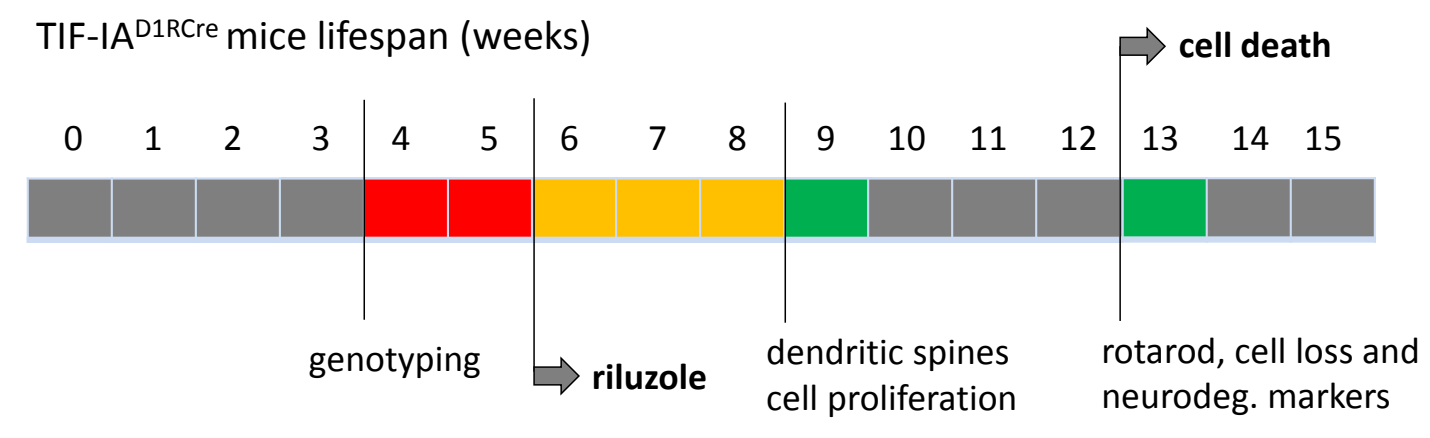


338 Fig. 2. Representative images of immunofluorescent analysis and quantification of proliferating 339 cells in the region of the SVZ as revealed by the Ki67 marker in control (con) and TIF-IA ${ }^{\text {D1RCre_ }}$ 340 mutant (mut) riluzole treated and non-treated mice (A, B). Example of Ki67/MAP2/DAPI (C) or $341 \mathrm{Ki67/DCX/DAPI} \mathrm{(D)} \mathrm{triple-stainings} \mathrm{carried} \mathrm{out} \mathrm{in} \mathrm{attempt} \mathrm{to} \mathrm{confirm} \mathrm{nuclear} \mathrm{localization} \mathrm{and}$ 342 neuronal origin of Ki67 signal $(\mathbf{C})$. Numbers of Ki67+ cells are represented by means \pm S.E.M. (n $343=4-6 ;{ }^{*} \mathrm{p}<0.05$ vs. con/VEH). RIL - riluzole, VEH - vehicle. Scale bars: $50 \mu \mathrm{m}$.
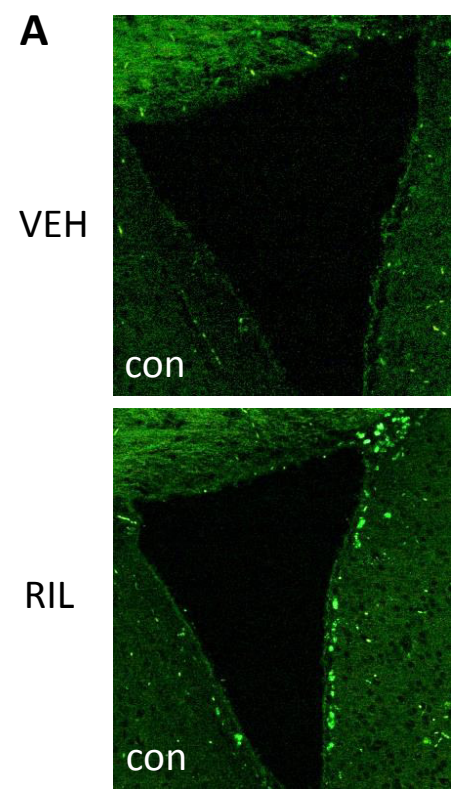

C

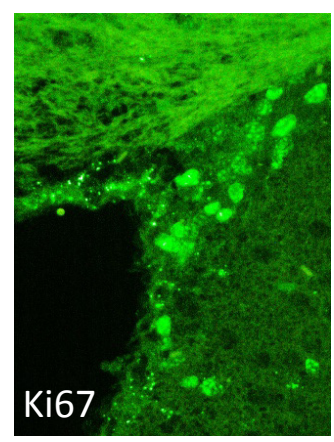

D

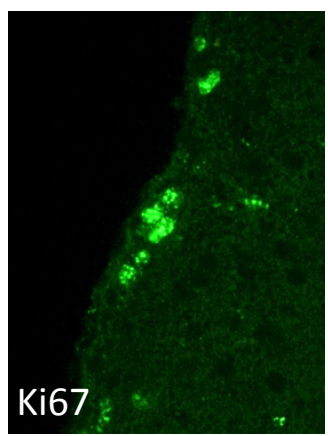

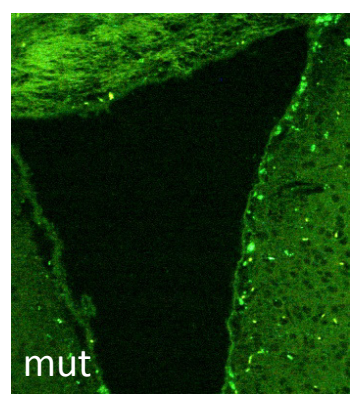
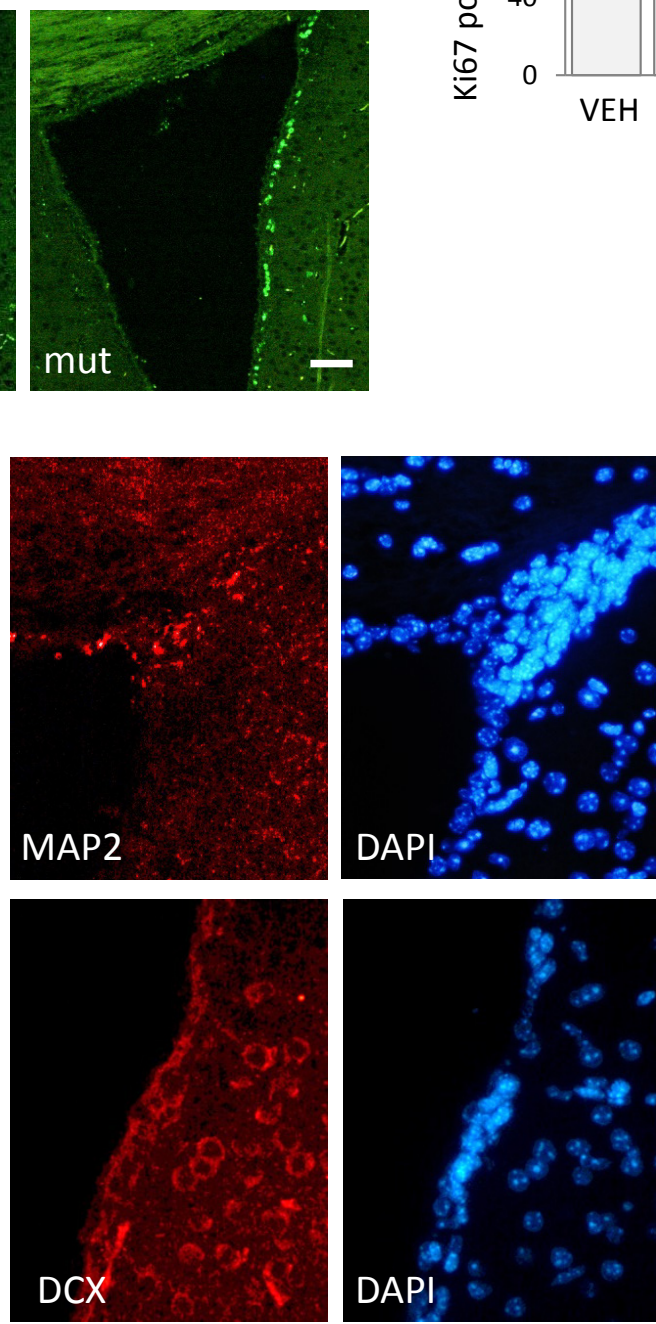

B
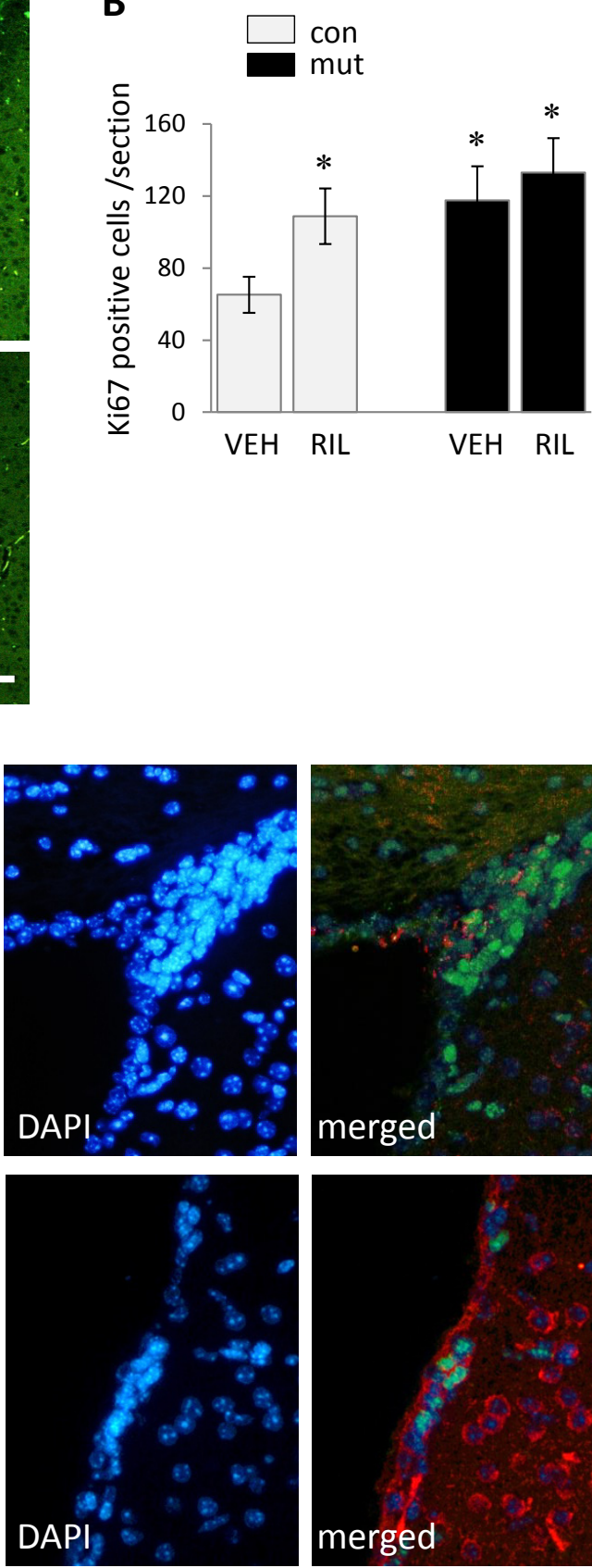
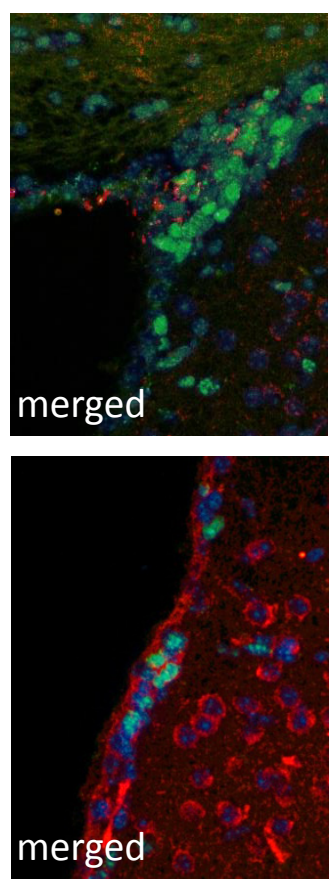
345 Fig. 3. Representative images of immunohistochemical analysis showing staining of striata with 346 the NeuN (A), induction of oxidative stress detected by the anti-8OHdG antibody (B), 347 astrogliosis visualized by the GFAP-specific antibody (C) and degenerating neurons detected by

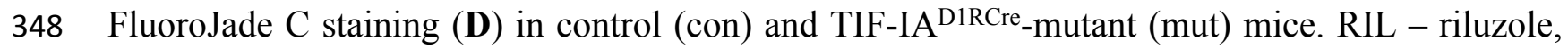
349 VEH - vehicle. Scale bars: $5 \mu \mathrm{m}(\mathbf{A}, \mathbf{C}, \mathbf{D}), 25 \mu \mathrm{m}(\mathbf{B})$.

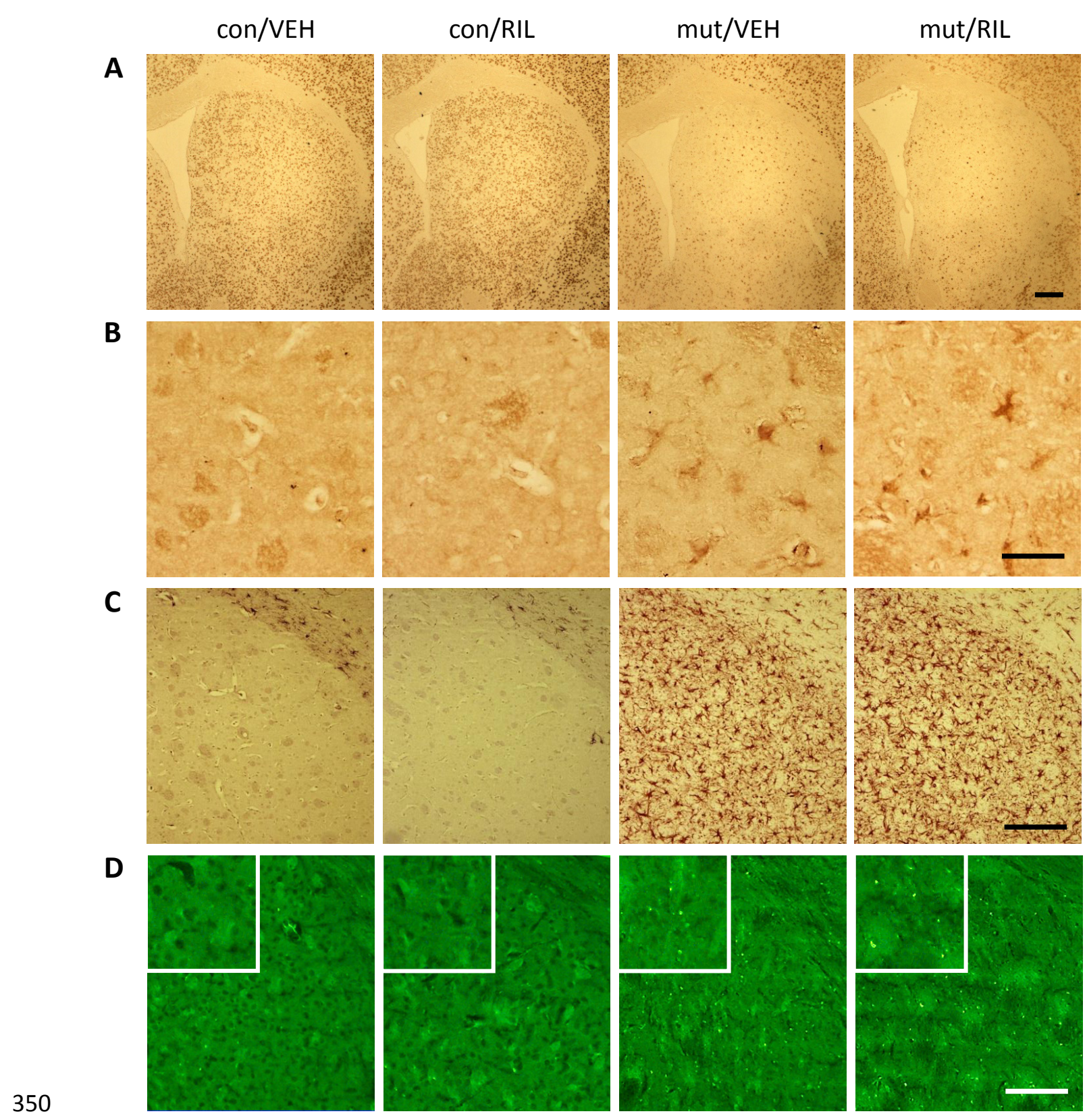


352 Fig. 4. Assessment of motor coordination of control (con) and TIF-IA ${ }^{\mathrm{D} 1 \mathrm{RCre}}$-mutant (mut) mice 353 demonstrated by endurance in the rotarod test. Values for endurance time are represented by 354 means \pm S.E.M. $(\mathrm{n}=7-8 ; * \mathrm{p}<0.05 ; * * \mathrm{p}<0.01$ vs. con/VEH). RIL - riluzole, VEH - vehicle.

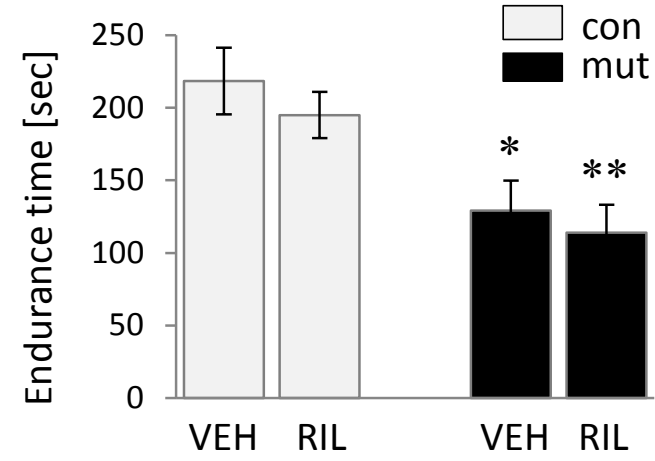

356 
357 Fig. 5. Visualization (A-B) and quantification (C) of dendritic spines in the control (con) and

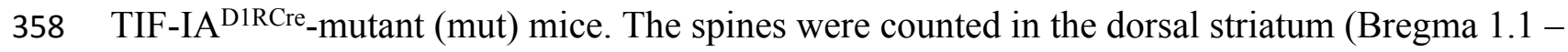
359 0.0). Data are represented by the means \pm SEM $(n=3-4)$. RIL - riluzole, VEH - vehicle.

A
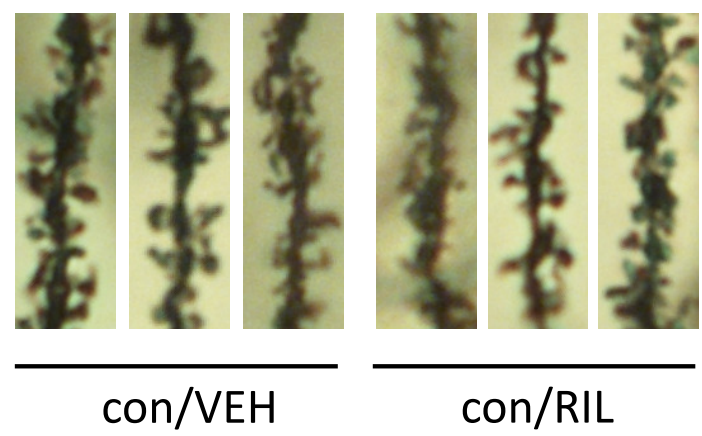

con/RIL

B

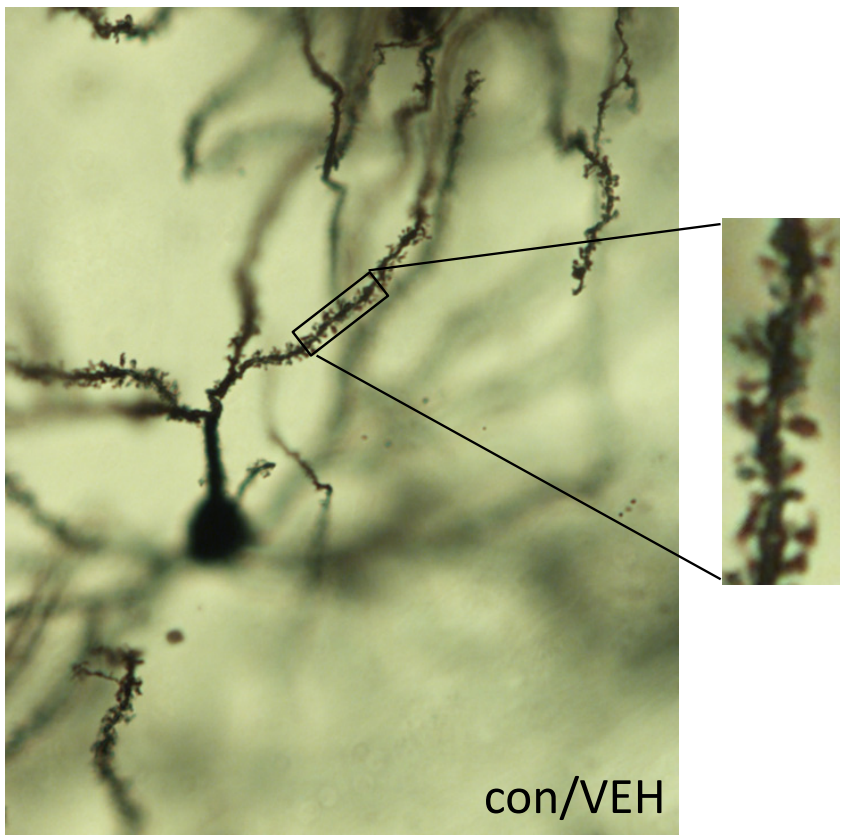

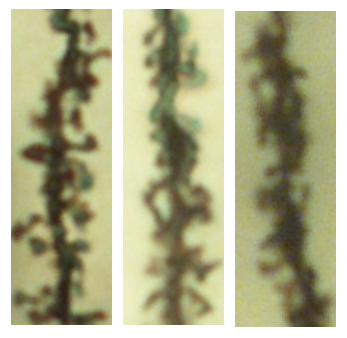

mut /VEH

C
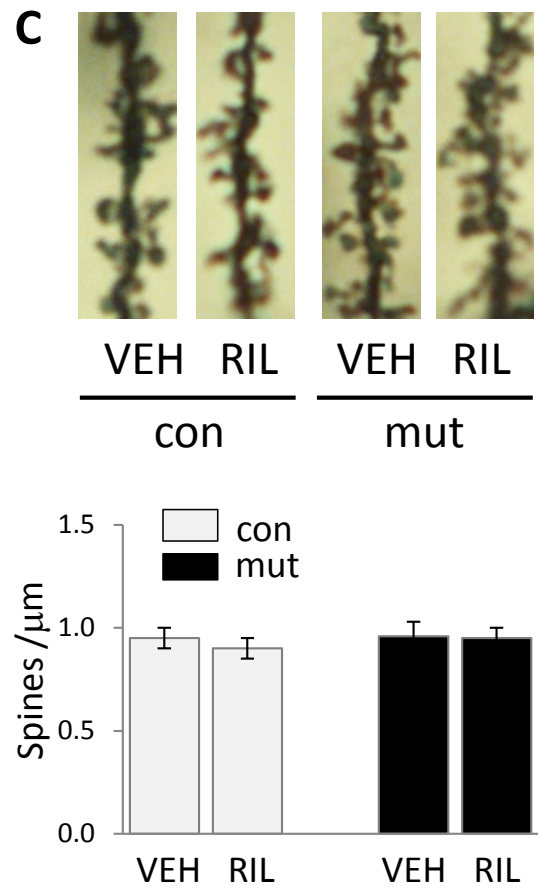


\section{References}

Abdipranoto A, Wu S, Stayte S, and Vissel B. 2008. The role of neurogenesis in neurodegenerative diseases and its implications for therapeutic development. CNS Neurol Disord Drug Targets $7: 187-210$.

Bergerot A, Shortland PJ, Anand P, Hunt SP, and Carlstedt T. 2004. Co-treatment with riluzole and GDNF is necessary for functional recovery after ventral root avulsion injury. Exp Neurol 187:359-366.

Besheer J, Lepoutre V, and Hodge CW. 2009. Preclinical evaluation of riluzole: assessments of ethanol self-administration and ethanol withdrawal symptoms. Alcohol Clin Exp Res 33:1460-1468.

Bonfanti L, and Peretto P. 2007. Radial glial origin of the adult neural stem cells in the subventricular zone. Prog Neurobiol 83:24-36.

Carbone M, Duty S, and Rattray M. 2012. Riluzole neuroprotection in a Parkinson's disease model involves suppression of reactive astrocytosis but not GLT-1 regulation. BMC Neurosci 13:38.

Chmielarz P, Kreiner G, Kot M, Zelek-Molik A, Kowalska M, Baginska M, Daniel WA, and Nalepa I. 2015. Disruption of glucocorticoid receptors in the noradrenergic system leads to BDNF upregulation and altered serotonergic transmission associated with a depressive-like phenotype in female GR(DBHCre) mice. Pharmacol Biochem Behav 137:69-77.

Chmielarz P, Kusmierczyk J, Parlato R, Schutz G, Nalepa I, and Kreiner G. 2013. Inactivation of glucocorticoid receptor in noradrenergic system influences anxiety- and depressive-like behavior in mice. PLoS One 8:e72632.

Dickstein DL, Brautigam H, Stockton SD, Jr., Schmeidler J, and Hof PR. 2010. Changes in dendritic complexity and spine morphology in transgenic mice expressing human wild-type tau. Brain Struct Funct 214:161-179.

Doble A. 1996. The pharmacology and mechanism of action of riluzole. Neurology 47:S233-241.

Emsley JG, Mitchell BD, Kempermann G, and Macklis JD. 2005. Adult neurogenesis and repair of the adult CNS with neural progenitors, precursors, and stem cells. Prog Neurobiol 75:321-341.

Frank S. 2014. Treatment of Huntington's disease. Neurotherapeutics 11:153-160.

Fumagalli E, Bigini P, Barbera S, De Paola M, and Mennini T. 2006. Riluzole, unlike the AMPA antagonist RPR119990, reduces motor impairment and partially prevents motoneuron death in the wobbler mouse, a model of neurodegenerative disease. Exp Neurol 198:114-128.

Gauthier LR, Charrin BC, Borrell-Pages M, Dompierre JP, Rangone H, Cordelieres FP, De Mey J, MacDonald ME, Lessmann V, Humbert S, and Saudou F. 2004. Huntingtin controls neurotrophic support and survival of neurons by enhancing BDNF vesicular transport along microtubules. Cell 118:127-138.

Ginsberg G, and Lowe S. 2002. Cost effectiveness of treatments for amyotrophic lateral sclerosis: a review of the literature. Pharmacoeconomics 20:367-387.

Hannan AJ. 2005. Novel therapeutic targets for Huntington's disease. Expert Opin Ther Targets 9:639650.

Hockly E, Tse J, Barker AL, Moolman DL, Beunard JL, Revington AP, Holt K, Sunshine S, Moffitt H, Sathasivam K, Woodman B, Wanker EE, Lowden PA, and Bates GP. 2006. Evaluation of the benzothiazole aggregation inhibitors riluzole and PGL-135 as therapeutics for Huntington's disease. Neurobiol Dis 21:228-236. 
404

405

406

407

408

409

410

411

412

413

414

415

416

417

418

419

420

421

422

423

424

425

426

427

428

429

430

431

432

433

434

435

436

437

438

439

440

441

442

443

444

445

446

Katoh-Semba R, Asano T, Ueda H, Morishita R, Takeuchi IK, Inaguma Y, and Kato K. 2002. Riluzole enhances expression of brain-derived neurotrophic factor with consequent proliferation of granule precursor cells in the rat hippocampus. FASEB J 16:1328-1330.

Kazantsev AG, and Hersch SM. 2007. Drug targeting of dysregulated transcription in Huntington's disease. Prog Neurobiol 83:249-259.

Kee N, Sivalingam S, Boonstra R, and Wojtowicz JM. 2002. The utility of Ki-67 and BrdU as proliferative markers of adult neurogenesis. J Neurosci Methods 115:97-105.

Kitzman PH. 2009. Effectiveness of riluzole in suppressing spasticity in the spinal cord injured rat. Neurosci Lett 455:150-153.

Kreiner G. 2015. Compensatory mechanisms in genetic models of neurodegeneration: are the mice better than humans? Front Cell Neurosci 9:56.

Kreiner G, Bierhoff H, Armentano M, Rodriguez-Parkitna J, Sowodniok K, Naranjo JR, Bonfanti L, Liss B, Schutz G, Grummt I, and Parlato R. 2013. A neuroprotective phase precedes striatal degeneration upon nucleolar stress. Cell Death Differ 20:1455-1464.

Limpert AS, Mattmann ME, and Cosford ND. 2013. Recent progress in the discovery of small molecules for the treatment of amyotrophic lateral sclerosis (ALS). Beilstein J Org Chem 9:717-732.

Lindvall O, and Kokaia Z. 2010. Stem cells in human neurodegenerative disorders--time for clinical translation? J Clin Invest 120:29-40.

Luzzati F, De Marchis S, Parlato R, Gribaudo S, Schutz G, Fasolo A, and Peretto P. 2011. New striatal neurons in a mouse model of progressive striatal degeneration are generated in both the subventricular zone and the striatal parenchyma. PLoS One 6:e25088.

Miller RG, Mitchell JD, and Moore DH. 2012. Riluzole for amyotrophic lateral sclerosis (ALS)/motor neuron disease (MND). Cochrane Database Syst Rev:CD001447.

Nato G, Caramello A, Trova S, Avataneo V, Rolando C, Taylor V, Buffo A, Peretto P, and Luzzati F. 2015. Striatal astrocytes produce neuroblasts in an excitotoxic model of Huntington's disease. Development 142:840-845.

Nithianantharajah J, Barkus C, Vijiaratnam N, Clement O, and Hannan AJ. 2009. Modeling brain reserve: experience-dependent neuronal plasticity in healthy and Huntington's disease transgenic mice. Am J Geriatr Psychiatry 17:196-209.

Parlato R, Kreiner G, Erdmann G, Rieker C, Stotz S, Savenkova E, Berger S, Grummt I, and Schutz G. 2008. Activation of an endogenous suicide response after perturbation of rRNA synthesis leads to neurodegeneration in mice. J Neurosci 28:12759-12764.

Rafa-Zabłocka K, Jurga A, Bagińska M, Parlato R, Schütz G, Nalepa I, and Kreiner G. 2014. Involvement of noradrenergic system in Parkinson's disease - study on novel transgenic mouse models. Eur Neuropsychopharmacol 24:S642-S643

Ransome MI, Renoir T, and Hannan AJ. 2012. Hippocampal neurogenesis, cognitive deficits and affective disorder in Huntington's disease. Neural Plast 2012:874387.

Rieker C, Engblom D, Kreiner G, Domanskyi A, Schober A, Stotz S, Neumann M, Yuan X, Grummt I, Schutz G, and Parlato R. 2011. Nucleolar disruption in dopaminergic neurons leads to oxidative damage and parkinsonism through repression of mammalian target of rapamycin signaling. $J$ Neurosci 31:453-460.

Sarnat HB. 2013. Clinical neuropathology practice guide 5-2013: markers of neuronal maturation. Clin Neuropathol 32:340-369. 
447

448

449

450

451

452

453

454

455

456

457

458

459

460

461

462

463

464

465

466

467

468

469

470

471

472

473

474

475
Schmidt J, Schmidt T, Golla M, Lehmann L, Weber JJ, Hubener-Schmid J, and Riess O. 2016. In vivo assessment of riluzole as a potential therapeutic drug for spinocerebellar ataxia type $3 . J$ Neurochem 138:150-162.

Schmued LC, Stowers CC, Scallet AC, and Xu L. 2005. Fluoro-Jade C results in ultra high resolution and contrast labeling of degenerating neurons. Brain Res 1035:24-31.

Sepulveda J, Astorga JG, and Contreras E. 1999. Riluzole decreases the abstinence syndrome and physical dependence in morphine-dependent mice. Eur J Pharmacol 379:59-62.

Simard JM, Tsymbalyuk O, Keledjian K, Ivanov A, Ivanova S, and Gerzanich V. 2012. Comparative effects of glibenclamide and riluzole in a rat model of severe cervical spinal cord injury. Exp Neurol 233:566-574.

Spires TL, Grote HE, Garry S, Cordery PM, Van Dellen A, Blakemore C, and Hannan AJ. 2004. Dendritic spine pathology and deficits in experience-dependent dendritic plasticity in R6/1 Huntington's disease transgenic mice. Eur J Neurosci 19:2799-2807.

Squitieri F, Ciammola A, Colonnese C, and Ciarmiello A. 2008. Neuroprotective effects of riluzole in Huntington's disease. Eur J Nucl Med Mol Imaging 35:221-222.

Tunez I, Tasset I, Perez-De La Cruz V, and Santamaria A. 2010. 3-Nitropropionic acid as a tool to study the mechanisms involved in Huntington's disease: past, present and future. Molecules 15:878916.

Verhave PS, Jongsma MJ, Van Den Berg RM, Vanwersch RA, Smit AB, and Philippens IH. 2012. Neuroprotective effects of riluzole in early phase Parkinson's disease on clinically relevant parameters in the marmoset MPTP model. Neuropharmacology 62:1700-1707.

Veyrac A, Sacquet J, Nguyen V, Marien M, Jourdan F, and Didier A. 2009. Novelty determines the effects of olfactory enrichment on memory and neurogenesis through noradrenergic mechanisms. Neuropsychopharmacology 34:786-795.

Yuan X, Zhou Y, Casanova E, Chai M, Kiss E, Grone HJ, Schutz G, and Grummt I. 2005. Genetic inactivation of the transcription factor TIF-IA leads to nucleolar disruption, cell cycle arrest, and p53-mediated apoptosis. Mol Cell 19:77-87. 